\title{
Astrocyte Swelling
}

National Cancer Institute

\section{Source}

National Cancer Institute. Astrocyte Swelling. NCI Thesaurus. Code C161540.

A morphologic finding indicating the presence of astrocytes with intracytoplasmic swelling. 